\title{
A Survey on Routing Protocols and its Issues in VANET
}

\author{
Jagadeesh Kakarla ${ }^{1}$, S Siva Sathya ${ }^{1}$, B Govinda Laxmi², Ramesh Babu B ${ }^{3}$ \\ ${ }^{1}$ School of Engineering and Technology, Department of Computer Science, Pondicherry University, \\ Pondicherry-India \\ ${ }^{2}$ Department of computer science, SSCE College, Andhra Pradesh-India \\ ${ }^{3}$ Department of computer science, Koneru Lakshmaiah University, Andhra Pradesh-India
}

\begin{abstract}
Vehicular ad-hoc networks (VANETs) offer a vast number of applications without any support from fixed infrastructure. These applications forward messages in a multi-hop fashion. Designing an efficient routing protocol for all VANET applications is very hard. Hence a survey on routing protocols based on various parameters of VANET is a necessary issue in vehicle-to- vehicle (V2V) and infrastructure-to- vehicle (IVC) communication. This paper gives a brief overview of different routing algorithms in VANET along with major classifications. The protocols are also compared based on their essential characteristics and tabulated.
\end{abstract}

\section{Keywords}

ZRP, HARP, VGPR, MIBR, DTSG, EAEP, CBLR, SRB, RAR.

\section{INTRODUCTION}

Vehicular Ad hoc Network (VANET) is considered as a special type of Mobile Ad hoc Network (MANET), which gains interest from many researchers. In VANET each vehicle acts as a router to exchange data between nodes in the network. It is designed for vehicle-to-vehicle (V2V) and infrastructure-to-vehicle (IVC) communication. Such networks are used in traffic control applications, safety applications, driver assistance and location based services. In VANETs power consumption and storage capacity are not limited and the position of the nodes can be determined by using GPS.

VANET has unique characteristics like high mobility with the constraint of road topology, initially low market penetration ratio, unbounded network size, infrastructure support that differentiate it from MANET. From the above mentioned characteristics, it is evident that conventional MANET routing protocols has difficulties from finding stable routing paths in VANET environments. Therefore, more and more researchers have concentrated on proposing suitable routing protocols to deal with the highly dynamic nature of VANET. The routing protocols in VANET are categorized into six types: Topology based, Position based, Geocast based, Cluster based, Broadcast based and Infrastructure based.

The rest of the paper is organized as follows. Section 2 deals with Topology based protocols, Section 3 on Position based protocols, Section 4 on Geocast based protocols, Section 5 on Cluster based protocols, Section 6 Broadcast based protocols and Section 7 briefs Infrastructure based protocols, Section 8 gives a comparison of the various routing protocols, Section 9 concludes the paper.

\section{Topology based protocols:}

These protocols discover the route and maintain it in a table before the sender starts transmitting data. They are further divided into Proactive, Reactive and hybrid protocols.

\subsection{Proactive protocols:}

The proactive protocol is also known as table driven routing protocol. These protocols work by periodically exchanging the knowledge of topology among all the nodes of the network. The proactive protocols do not have initial route discovery delay but consumes lot of bandwidth for periodic updates of topology. There are several routing protocols that fall under this category.

Fisheye state routing (FSR) [3]: It is similar to link state routing protocol (LSR). Each node maintains a topology table based on the latest information received from neighborhood nodes. It uses different exchange period for different entries in routing table to reduce the size of control messages in large networks. The disadvantage in FSR routing, is the size of the routing table increases with increase in network size. Route discovery may fail if the destination node lies out of scope of source node. Due to high mobility in VANET, route to remote destination become less accurate.

Optimized Link State Routing Protocol (OLSR) [5]: It is an optimization of a pure link state protocol for mobile ad hoc networks. Each node in the network selects a set of neighbor nodes called as multipoint relays (MPR) which retransmits its packets. The neighbor nodes which are not in its MPR set can only read and process the packet. This procedure reduces the number of retransmissions in a broadcast procedure.

Topology Dissemination Based on Reverse-Path Forwarding (TBRPF) [8]: It is a link-state routing protocol designed for ad-hoc networks. Every node constructs a source tree which contains paths to all reachable nodes by using topology table. Nodes are periodically updated with only the differences between the previous and current network state using HELLO messages. Therefore, routing messages are smaller, can therefore be sent more frequently to neighbors.

\subsection{Reactive protocols:}

These protocols are called as on-demand routing protocols as they periodically update the routing table, when some data is there to send. But these protocols use flooding process for route discovery, which causes more routing overhead and also suffer from the initial route discovery process, which make them unsuitable for safety applications in VANET. 
$\boldsymbol{A O D V}$ [2]: Is a source initiated routing protocol and uses HELLO messages to identify its neighbors. Source node broadcasts a route request to its neighbors which fill forward to the destination. Then the destination unicast a route reply packet to the sender. Every node maintains broadcast-id which increments for new RREQ. when a RREQ arrives at a node, it checks the broadcast id if it is less than or equal to previous message then it will discard the packet.

$\boldsymbol{D S R}$ [1]: It Uses source routing instead of depending on intermediate node routing table. So routing overhead is always dependent on the path length. The limitation of this protocol is that the route maintenance process does not locally repair a broken link. The performance of the protocol briskly decreases with increasing mobility.

Temporally-Ordered Routing Algorithm (TORA) [15]: Each node constructs a directed cyclic graph by broadcasting query packets. On receiving a query packet, if the node has a route to destination it will send a reply packet, else it drops the packet. A node on receiving a reply packet will update its height only if the height of packet is minimum than other reply packets. It gives a route to all the nodes in the network, but the maintenance of all these routes is difficult in VANET.

\subsection{Hybrid protocol:}

The hybrid protocols are introduced to reduce the control overhead of proactive routing protocols and decrease the initial route discovery delay in reactive routing protocols.

\section{Zone routing protocol ( $\mathrm{ZRP})$ [24]:}

In this the network is divided into overlapping zones. The zone is defined as a collection of nodes which are in a zone radius. The size of a zone is determined by a radius of length $\alpha$ where $\alpha$ is the number of hops to the perimeter of the zone. In ZRP, a proactive routing protocol (IARP) is used in intra-zone communication and an inner-zone reactive routing protocol (IARP) is used in intra-zone communication. Source sends data directly to the destination if both are in same routing zone otherwise IERP reactively initiates a route discovery. ZRP aims to find loop free routes to the destination. It uses bordercasting method to construct multicast trees to flood the query packets instead of standard flooding to discover the destination route.

$\boldsymbol{H A R P}$ [17]: It divides entire network into non-overlapping zones. It aims to establish a stable route from a source to a destination to improve delay. It applies route discovery between zones to limit flooding in the network, and choose best route based on the stability criteria. In HARP routing is performed on two levels: intra-zone and inter-zone, depending on the position of destination. It uses proactive and reactive protocols in intrazone and inter-zone routing respectively. It is not applicable in high mobility adhoc networks.

\section{Position based protocols:}

These protocols use geographic positioning information to select the next forwarding hops so no global route between source and destination needs to be created and maintained.
Greedy Perimeter Stateless Routing (GPSR) [4]: Each node periodically broadcasts a beacon message to all its neighbors containing its id and position. If any node does not receives any beacon message from a neighbor for a specific period of time, then GPSR router assumes that the neighbor has failed or out of range, and deletes the neighbor from its table. It takes greedy forwarding decisions using information about immediate neighbors in the network. For any node if greedy forwarding is impossible then it uses perimeter of the region strategy to find the next forwarding hop. In a city scenario greedy forwarding is often restricted because direct communications between nodes may not exist due to obstacles such as buildings and trees. Converting network topology into planarized graph when greedy forwarding is not possible will degrade the performance of routing.

The authors in [23] eliminated graph planarization in Greedy Perimeter Coordinator Routing (GPCR) it consists of two parts: a restricted greedy forwarding procedure and a repair strategy which is based on the topology of real world streets and junctions and hence does not require a graph planarization process. The GPCR takes advantage of the fact that streets and junctions form a natural planar graph, without using any static street map.

\section{Vertex-Based predictive Greedy Routing $($ VGPR) [6]:}

It is a multi-hop vehicle-to-infrastructure routing protocol for urban environment. It estimates a sequence of valid junctions from a source node to fixed infrastructure and then, transmit message to the fixed infrastructure through the sequence of junctions. It uses position, velocity and direction of vehicles for calculating both sequence of valid junctions and greedy forwarding. In calculation of a sequence of valid junctions, a source node calculates the shortest path between itself and its nearest fixed infrastructure with the help of navigation system. If the source node gets more than one route to the fixed infrastructure with same number of junctions then it randomly selects one route among them. It uses predictive directional greedy routing (PDGR) [28] to forward data from source node to the nearest fixed infrastructure. Each vehicle maintains a table, containing id, position, velocity and direction of its two-hop neighbors. The table is periodically updated by exchanging beacon messages among neighboring vehicles. With the help of a table, the source node calculates the weighted score for itself, current packet carrier and for two-hop neighbors. If a neighbor has the higher score than the current packet carrier, the source vehicle forwards the packet to the neighbor otherwise, the current packet carrier carries the packet until it finds its neighbor which has higher weighted score than itself. VGPR have less control overhead, reduces packet retransmissions, increases reliability of packet delivery, and minimizes end to end delay.

MIBR [22]: It uses buses as a key element in route selection and data transfer process. While designing the protocol quality of transmission for each road segment and different transmission abilities of various vehicles are also considered. It measures the density of every road segment using bus line information. MIBR is a location based reactive routing protocol. Source node uses GPS system to get the destination information. Each bus 
contains two heterogeneous wireless interfaces and other vehicles have single interface. While routing it estimates next road segment and hop count and stored in a route table. The next road segment is chosen when the packet is near a junction. This process consumes less bandwidth. In packet forwarding process it uses "bus first" strategy. MIBR is only suitable in urban scenarios.

$\boldsymbol{G Y T A R}$ [26]: Is an improved Greedy Traffic Aware Routing Protocol for Vehicular Ad Hoc Networks in City Environments. It contains two modules: Junctions selection, forwarding data between two junctions. A packet will pass through junctions to reach its destination. In junction selection process a value is given to each junction by comparing the traffic density between the current junction and the next candidate junction and the curvemetric distance to the destination. The junction with highest value will be chosen for packet forwarding. In second module each vehicle maintains a table which contains position, velocity and direction of each neighbor vehicle and the table is updated periodically. Thus, when a packet is received, the forwarding vehicle computes the new predicted position of each neighbor using the table and then selects the next hop neighbor which is closer to the destination junction which may cause packets in a local optimum. To overcome this problem GYTAR uses store and forward strategy. In this strategy packet will be stored at the intermediate node until another vehicle which is closer to the destination junction enters in its transmission range. Due to high mobility in VANET all greedy forwarding protocols can also cause routing loops problem and some packets may get forwarded to the wrong direction.

\section{Geocast based protocols:}

These protocols are used to send a message to all vehicles in a pre-defined geographical region.

Robust Vehicular Routing (ROVER) [9]: It is a reliable geographical multicast protocol where only control packets are broadcasted in the network and the data packets are unicasted. The objective of the protocol is to send a message to all other vehicles within a specified Zone of Relevance (ZOR). The ZOR is defined as a rectangle specified by its corner coordinates. A message is defined by the triplet $[\mathrm{A}, \mathrm{M}, \mathrm{Z}]$ it indicates specified application, message and identity of a zone respectively. When a vehicle receives a message, it accepts the message if it is within the ZOR. It also defines a Zone of Forwarding (ZOF) which includes the source and the ZOR. All vehicles in the $\mathrm{ZOF}$ are used in the routing process. It uses a reactive route discovery process within a ZOR. This protocol creates lot of redundant messages in the network which leads to congestion and high delay in data transfer. To overcome this problem authors in [25] proposed a Two Zone Dissemination Protocol for VANET. It uses hop-count in packet and is decremented when the packet is forwarded. If the hop-count reaches to zero, the packet will be discarded. It causes nodes near to the sender forward a packet multiple times. To avoid it they introduced sequence number for every packet to detect whether a packet has been received before or not.

$\boldsymbol{D T S G}$ [13]: The main aim of this protocol is to work even with sparse density networks. It dynamically adjusts the protocol depending on network density and the vehicles speed for better performance. It defines two phases: pre-stable and stable period. Pre-stable phase helps the message to be disseminated within the region, and stable-period intermediate node uses store and forward method for a predefined time within the region. It also tries to balance between packet delivery ratio and network cost.

\section{Cluster based protocols:}

In Cluster-based routing protocols vehicles near to each other form a cluster. Each cluster has one cluster-head, which is responsible for intra and inter-cluster management functions. Intra-cluster nodes communicate each other using direct links, whereas inter-cluster communication is performed via clusterheaders. In cluster based routing protocols the formation of clusters and the selection of the cluster-head is an important issue. In VANET due to high mobility dynamic cluster formation is a towering process.

$\boldsymbol{H C B}$ [19]: It is a novel based Hierarchical Cluster routing protocol designed for highly mobility adhoc networks. HCB is two-layer communication architecture. In layer-1 mostly nodes have single radio interface and they communicate with each other via multi-hop path. Among these nodes some also have another interface with long radio communication range called super nodes which exist both on layer-1and 2. Super nodes are able to communicate with each other via the base station in layer-2. During the cluster formation, each node will attach to the nearest cluster header and super nodes will become cluster headers in layer-1. In HCB, intra-cluster routing is performed independently in each cluster. Cluster heads exchange membership information periodically to enable inter-cluster routing.

Cluster Based Location Routing (CBLR) [11]: It is a reactive and cluster based routing protocol. In cluster formation every node broadcasts a hello message and waits for a predefined time. If the node receives a reply message from a cluster header before the timer expires, it becomes a cluster member. Otherwise, it becomes a cluster header. Each cluster header maintains a table contains the addresses and geographic locations of the cluster members and gateways nodes, and it also maintains a Cluster Neighbor Table that contains information about the neighboring clusters. When a source wants to send data to a destination, it first checks whether the destination is in the same cluster or not. If it is in same cluster, it sends the packet to the closest neighbor to the destination. Otherwise, the source stores the data packet in its buffer, starts a timer and broadcasts Location Request (LREQ) packets. Only gateways and cluster-heads can retransmit the LREQ packet to minimize number of retransmissions. After receiving a request, each cluster-head checks whether the destination is a member of its cluster or not. If it is a cluster member, then cluster header sends a Location Reply (LREP) packet to the sender based on the information in LREQ packet and cluster neighbor table. Otherwise it retransmits to adjacent cluster-headers. CBLR is suitable for high mobility networks because it updates the location of the source and destination every time before data transmission starts.

Cluster Based Routing (CBR) [16]: The geographic area is divided into square grids. Each node calculates optimal neighbor cluster header to forward data to the next hop by using 
geographic information. The routing overhead is less as it need not discover route and save in routing table. The cluster header broadcasts a LEAD message to its neighbors with coordinate of its grid and the location of cluster header. If there is a road side unit (RSU) in the grid it will become a cluster header. Whenever the header is leaving the grid, it will broadcast LEAVE message containing its grid position. An intermediate node stores it until a new cluster header is selected. The new cluster header uses this information for data routing. This protocol does not consider velocity and direction which are important parameters in VANET.

\section{Cluster-Based Directional Routing Protocol} (CBDRP) [27]: It divides the vehicles into clusters and vehicles which are moving in same direction form a cluster. The source sends the message to its cluster header and then it forwards the message to header which is in the same cluster with the destination. At last the destination header sends the message to the destination. The cluster header selection and maintenance is same like CBR but it considers velocity and direction of a vehicle.

\section{Broadcast based protocols:}

Edge-aware epidemic protocol (EAEP) [14]: It is reliable, bandwidth efficient information dissemination based highly dynamic VANET protocol. It reduces control packet overhead by eliminating exchange of additional hello packets for message transfer between different clusters of vehicles and eases cluster maintenance. Each vehicle piggybacks its own geographical position to broadcast messages to eliminate beacon messages. Upon receiving a new rebroadcast message, EAEP uses number of transmission from front nodes and back nodes in a given period of time to calculate the probability for making decision whether nodes will rebroadcast the message or not. But EAEP does not address the intermittent connectivity issue. Specifically, a node does not know whether it has missed any messages to its new neighbors or its neighbors have missed some messages. EAEP overcomes the simple flooding problem but it incurs high delay of data dissemination.

\section{Distributed vehicular broadcast protocol (DV-} $\boldsymbol{C A S T ) ~ [ 1 8 ] : ~ I t ~ u s e s ~ l o c a l ~ t o p o l o g y ~ i n f o r m a t i o n ~ b y ~ u s i n g ~ t h e ~}$ periodic hello messages for broadcasting the information. Each vehicle uses a flag variable to check whether the packet is redundant or not. This protocol divides the vehicles into three types depending on the local connectivity as well connected, sparsely connected, totally disconnected neighborhood. In well connected neighborhood it uses persistence scheme (weighted ppersistence, slotted 1and $\mathrm{p}$ persistence). In sparsely connected neighborhood after receiving the broadcast message, vehicles can immediately rebroadcast with vehicles moving in the same direction. In totally disconnected neighborhood vehicles are used to store the broadcast message until another vehicle enters into transmission range, otherwise if the time expires it will discard the packet. This protocol causes high control overhead and delay in end to end data transfer.

Secure Ring Broadcasting (SRB) [10]: It is to minimize number of retransmission messages and to get more stable routes. It classifies nodes into three groups based on their receiving power as Inner Nodes (close to sending node), Outer
Nodes (far away from sending node), Secure Ring Nodes (preferable distance from sending node). It restricts rebroadcasting to only secure ring nodes to minimize number of retransmissions.

$\boldsymbol{P B S M}$ [21]: It is an adaptive broadcasting protocol that does not require nodes to know about position and movement of their nodes and itself. It uses connected dominating sets (CDS) and neighbor elimination concepts to eliminate redundant broadcasting. It employs two-hop neighbor information obtained by periodic beacons to construct CDS. Each vehicle A maintains two lists of neighboring vehicles: $\mathrm{R}$ and NR, containing neighbors that already received and that which did not receive the packet. After a timeout, A rebroadcasts the packet if the list $\mathrm{NR}$ is nonempty. Both lists $\mathrm{R}$ and NR are updated periodically by using beacon messages. Nodes in CDS have less waiting timeout than nodes that are not in CDS. The main idea of PBSM is two nodes do not transmit every time they discover each other as new neighbors. It is a parameter less protocol which does not consider vehicle position, direction and velocity. To overcome this problem authors proposed ACKPBSM [7] which tries to reduce the control packet overhead in data forwarding. It uses GPS to retrieve position information and acknowledgements are piggybacked in periodic beacon messages. It employs 1-hop position information obtained by periodic beacons to construct CDS.

As PBSM AND ACKPBSM uses store and forward method to deliver the message in whole network which employs high end to end delay this is not acceptable in safety application for VANET.

\section{Infrastructure based protocols:}

The following protocols are infrastructure based because the relay on fixed infrastructure for their routing.

$\boldsymbol{S A D V}$ [20]: Is a static node assisted adaptive data dissemination protocol for vehicular networks. It uses static nodes at junctions to forward a packet. It employs store and forward mechanism in the static nodes till a vehicle enters into transmission range or optimal path is available.

Roadside-Aided Routing (RAR) [12]: Is a framework for effective routing in vehicular hybrid networks rather than a concrete routing protocol. Here roads are divided into sectors by using road side units (RSUs), and the route consists of vehicles and RSUs. These protocols are not efficient in high way scenarios as they require static node or RSU.

\section{COMPARSION OF ROUTING PROTOCOLS}

The various protocols are compared based on important parameters and tabulated below. 


\begin{tabular}{|c|c|c|c|c|c|c|c|c|}
\hline Parameters & $\begin{array}{l}\text { Forwarding } \\
\text { Strategy }\end{array}$ & $\begin{array}{l}\text { Routing } \\
\text { Maintenance }\end{array}$ & Scenario & $\begin{array}{l}\text { Recovery } \\
\text { strategy }\end{array}$ & $\begin{array}{l}\text { Infrastructure } \\
\text { Require-ment }\end{array}$ & $\begin{array}{l}\text { Digital } \\
\text { map }\end{array}$ & $\begin{array}{l}\text { Control } \\
\text { Packet } \\
\text { overhead }\end{array}$ & $\begin{array}{l}\text { No of } \\
\text { retransmis } \\
\text { sion }\end{array}$ \\
\hline $\begin{array}{l}\text { FSR } \\
\end{array}$ & Multi hop & Proactive & Urban & Multi hop & No & No & High & Less \\
\hline OLSR & Multi hop & Proactive & Urban & Multi hop & No & No & High & Less \\
\hline TBRPF & Multi hop & Proactive & Urban & Multi hop & No & No & High & Less \\
\hline AODV & Multi hop & Reactive & Urban & $\begin{array}{l}\text { Store and } \\
\text { Forward }\end{array}$ & No & No & Low & Less \\
\hline DSR & Multi hop & Reactive & Urban & $\begin{array}{l}\text { Store and } \\
\text { forward }\end{array}$ & No & No & Low & Less \\
\hline TORA & Multi hop & Reactive & Urban & $\begin{array}{l}\text { Store and } \\
\text { forward }\end{array}$ & No & No & Low & Less \\
\hline ZRP & Multi hop & Hybrid & Urban & Multi hop & No & No & Moderate & Less \\
\hline HARP & Multi hop & Hybrid & Urban & Multi hop & No & No & Moderate & Less \\
\hline GPSR & $\begin{array}{l}\text { Greedy } \\
\text { forwarding }\end{array}$ & Reactive & Urban & $\begin{array}{l}\text { Store and } \\
\text { forward }\end{array}$ & No & Yes & Moderate & Less \\
\hline VGPR & $\begin{array}{l}\text { Greedy } \\
\text { forwarding }\end{array}$ & Reactive & Urban & $\begin{array}{l}\text { Store and } \\
\text { forward }\end{array}$ & No & Yes & Moderate & Less \\
\hline GPCR & $\begin{array}{l}\text { Greedy } \\
\text { Forwarding }\end{array}$ & Reactive & Urban & $\begin{array}{l}\text { Store and } \\
\text { forward }\end{array}$ & No & Yes & Moderate & Less \\
\hline MIBR & Bus first & Reactive & Urban & $\begin{array}{l}\text { Store and } \\
\text { forward }\end{array}$ & No & Yes & Low & Moderate \\
\hline GYTAR & $\begin{array}{l}\text { Greedy } \\
\text { forwarding }\end{array}$ & Reactive & Urban & $\begin{array}{l}\text { Store and } \\
\text { forward }\end{array}$ & No & Yes & Moderate & Less \\
\hline ROVER & Multi hop & Reactive & Urban & Flooding & No & No & High & High \\
\hline TZDP & Multi hop & Reactive & Urban & Flooding & No & No & Low & High \\
\hline DTSG & Multi hop & Reactive & Urban & Flooding & No & No & Moderate & High \\
\hline HCB & Multi hop & Reactive & Urban & $\begin{array}{l}\text { Store and } \\
\text { forward }\end{array}$ & No & Yes & Moderate & High \\
\hline CBLR & Multi hop & Reactive & Urban & Flooding & No & Yes & Less & High \\
\hline CBR & Multi hop & Reactive & Urban & $\begin{array}{l}\text { Store and } \\
\text { forward }\end{array}$ & No & Yes & Moderate & High \\
\hline CBDRP & Multi hop & Reactive & Urban & Store and & No & Yes & Moderate & High \\
\hline
\end{tabular}




\begin{tabular}{|c|c|c|c|c|c|c|c|c|}
\hline & & & & forward & & & & \\
\hline EAEP & Multi hop & Reactive & High way & $\begin{array}{l}\text { Store and } \\
\text { forward }\end{array}$ & No & No & High & Moderate \\
\hline DV-CAST & Multi hop & Proactive & High way & $\begin{array}{l}\text { Store and } \\
\text { forward }\end{array}$ & No & No & High & Moderate \\
\hline SRB & Multi hop & Reactive & High way & $\begin{array}{l}\text { Store and } \\
\text { forward }\end{array}$ & No & No & High & Moderate \\
\hline PBSM & Multi hop & Reactive & High way & $\begin{array}{l}\text { Store and } \\
\text { forward }\end{array}$ & No & No & High & Moderate \\
\hline ACKPBSM & Multi hop & Reactive & High way & $\begin{array}{l}\text { Store and } \\
\text { forward }\end{array}$ & No & No & High & Moderate \\
\hline SADV & $\begin{array}{l}\text { Store and } \\
\text { forward }\end{array}$ & Reactive & Urban & Multi hop & yes & No & Low & Low \\
\hline RAR & $\begin{array}{l}\text { Store and } \\
\text { forward }\end{array}$ & Reactive & Urban & Multi hop & Yes & No & Low & Low \\
\hline
\end{tabular}

\section{CONCLUSION:}

Routing is an important component in vehicle-to-vehicle (V2V) and infrastructure-to-vehicle (I2V) communication. This paper discusses various routing protocols of VANET. Designing an efficient routing protocol for all VANET applications is very hard. Hence a survey of different VANET protocols, comparing the various features is absolutely essential to come up with new proposals for VANET. The performance of VANET routing protocols depend on various parameters like mobility model, driving environment and many more. Thus this paper has come up with an exhaustive survey and comparison of different classes of VANET routing protocols. From the survey it is clear that position based, geocast and cluster based protocols are more reliable for most of the applications in VANET.

\section{REFERENCES}

[1] D. Johnson, B.D.A. Maltz, and Y.C.Hu, "The Dynamic Source Routing Protocol for Mobile Ad Hoc Networks (DSR)", draft-ietf-manet-dsr-10.txt, 2004.

[2] C. Perkins, E. Belding-Royer, and S. Das, "Ad Hoc OnDemand Distance Vector (AODV) Routing”, RFC 3561, Network Working Group, 2003.

[3] M. Gerla, X. Hong, G. Pei, "Fisheye State Routing Protocol (FSR)", IETF Internet Draft, work in progress, draft-ietfmanet-fsr-03.txt, July 2002.

[4] B. Karp and H.T. Kung, "GPSR: Greedy perimeter stateless routing for wireless networks", in Proceedings of the ACM/IEEE International Conference on Mobile Computing and Networking (MobiCom), 2000.
[5] T. Clausen, et al., "Optimized Link State Routing Protocol (OLSR)”, RFC 3626, Network Working Group, Oct. 2003.

[6] Raj K. Shrestha, Sangman Moh, Ilyong Chung, and Dongmin Choi, "Vertex-based multi-hop vehicle-toinfrastructure routing for vehicular adhoc networks", IEEE proceedings of 43rd Hawaii International Conference on System Sciences (HICSS) 2010.

[7] F. Ros, P.M. Ruiz, and I. Stojmenovic, "Reliable and efficient broadcasting in vehicular ad hoc networks", IEEE the 69th Vehicular Technology Conference (VTC'09), Spain, April, 2009.

[8] R. Ogier, et al., "Topology Dissemination Based on ReversePath Forwarding (TBRPF)", RFC 3684, Network Working Group, Feb 2004.

[9] M. Kihl, M. Sichitiu, T. Ekeroth and M. Rozenberg, "Reliable Geographical Multicast Routing in Vehicular Adhoc Networks" , Lecture Notes in Computer Science 4517 LNCS[C], pp. 315-325, 2007.

[10] Rainer Baumann, "Vehicular Ad hoc Networks", Master's Thesis in Computer Science, ETH Zurich 2004.

[11] R. A. Santns, R. M. Edwards, A. Edwards and D. Belis, "A novel cluster-based location routing algorithm for intervehicular communication", Personal, Indoor and Mobile Radio Communications, IEEE proceedings of the $15^{\text {th }}$ Annual Symposium, 2004.

[12] Y. Peng, Z. Abichar, and J. M. Chang," Roadside-aided routing(RAR) in vehicular networks", ICC'06 IEEE International Conference on Communications, 8:36023607, June 2006. 
[13] Hamidreza Rahbar, Kshira sagar Naik, Amiya Nayak, "DTSG: Dynamic Time-Stable Geocast Routing in Vehicular Ad Hoc Networks", IEEE Symposium on Computers and Communications, pp. 198-203, 2001.

[14] M. Nekovee, B. BjamiBogason, "Reliable and efficient information dissemination in intermittently connected vehicular ad hoc networks", IEEE the 65th VTC'07 spring, Dublin, Ireland, April 22-25, 2007.

[15] V. Park, S. Corson, "Temporally-Ordered Routing Algorithm (TORA) Version 1 Functional Specification", IETF Internet draft, work in progress, draft-ietf-manet-toraspec-04.txt, 2001.

[16] Yuyi Luo, Wei Zhang, Yangqing Hu, "A New Cluster Based Routing Protocol for VANET", IEEE Wireless Communications and Trusted Computing, 2010.

[17] Navid Nikaein, Christian Bonnet and Neda Nikaein, "HARP - Hybrid Ad Hoc Routing Protocol", in proceeding of IST 2001: International Symposium on Telecommunications, Iran/Tehran 2001.

[18] O. K. Tonguz, N. Wisitpongphan, F. Bai, P. Mudalige and V. Sadekar, "Broadcasting in VANET", Proc. IEEE INFOCOM MOVE Workshop 2007, Anchorage, USA, May, 2007.

[19] Yang Xia, Chai Kiat Yeo, Bu Sung Lee, " Hierarchical Cluster Based Routing for HighlyMobile Heterogeneous MANET", Communications and Information Technologies, ISCIT'07, International Symposium on, pp. 936-941, Oct 2007.

[20] Y. Ding, C.Wang, and L. Xiao, "A static-node assisted adaptive routing protocol in vehicular networks", VANET'07, Proceedings of the fourth ACM international workshop on Vehicular ad hoc networks, pages 59-68, September 2007.
[21] Adnan Afsar Khan, Ivan Stojmenovic, Nejib Zaguia, "Parameter less broadcasting in static to highly mobile wireless ad hoc, sensor and actuator networks", in Proc. ACM Int.Conference on Mobile Computing and Networking (MobiCom), Seattle, USA, August 1999.

[22] Jie Luo, Xinxing Gu, Tong Zhao, Wei Yan, “A Mobile Infrastructure Based VANET Routing Protocol in the Urban Environment", IEEE Conference on Communications and Mobile Computing, 2010.

[23] C. Lochert, M. Mauve, H. Fubler, and H. Hartenstein, "Geographic routing in city scenarios", ACM SIGMOBILE Mobile Computing and Communications Review (MC2R) [C], vol.9, no.1, pp. 69-72, Jan 2005.

[24] Z. J. Haas, "The Zone Routing Protocol (ZRP) for ad hoc networks", Internet Draft, Nov. 1997.

[25] Jeppe Bronsted and Lars Michael Kristensen, "Specification and Performance Evaluation of Two Zone Dissemination Protocols for Vehicular Ad-hoc Networks", IEEE Proceedings of the 39th Annual Simulation Symposium (ANSS'06).

[26] Moez Jerbi, Rabah Meraihi, Sidi-Mohammed Senouci, Yacine Ghamri-Doudane ENSIIE," GyTAR: improved Greedy Traffic Aware Routing Protocol for Vehicular Ad Hoc Networks in City Environments", VANET'06, September 2006.

[27] Tao Song, Wei Xia, Tiecheng Song, Lianfeng Shen,"A Cluster-Based Directional Routing Protocol in VANET", International Conference on Communication and Mobile Computing, 2010.

[28] J. Gong, C. Z. Xu, and J. Holle, "Predictive Directional Greedy Routing in Vehicular Ad Hoc Networks", Proc. of 27th International Conference on Distributed Computing Systems, 2007. 